\title{
EMBEDDINGS OF INFINITE PERMUTATION GROUPS IN SHARP, HIGHLY TRANSITIVE, AND HOMOGENEOUS GROUPS
}

\author{
by S. A. ADELEKE \\ (Received 4th February 1986, revised 9th April 1987)
}

\section{Introduction}

This paper answers two questions posed by P. M. Neumann and K. Hickin on embeddings of infinite permutation groups. We first give some definitions that would make their questions understood.

Definitions. Let $\Omega$ be an infinite set and let $G$ be a group such that $G \subseteq \operatorname{Sym}(\Omega)$, the set of all permutations of $\Omega$.

(i) $(G, \Omega)$ is said to be sharp if for every $g$ in $G \backslash\{1\}$, the set fix $(g)$ of all elements fixed by $g$ is empty or finite.

(ii) $(G, \Omega)$ is said to be highly transitive if for every natural number $m$ and every pair of finite sequences of $m$ distinct points in $\Omega:\left(\alpha_{1}, \ldots, \alpha_{m}\right)$ and $\left(\beta_{1}, \ldots, \beta_{m}\right)$, there exists $g$ in $G$ such that $\alpha_{i} g=\beta_{i}$ for all natural numbers $i$ less than or equal to $m$.

(iii) $(G, \Omega)$ is relatively homogeneous if for every finitely generated subgroup $B \subseteq G$, and every $B$-isomorphism $f$ among finitely many orbits of $B$, there exists $x$ in the centralizer $C_{G}(B)$ which extends $f$.

Other terms not defined here can be checked in one of the many books on Infinite Groups.

The questions and answers are:

Question 1 (P. M. Neumann). Is every countable sharp group acting on a countably infinite set a subgroup of a sharp highly transitive group acting on the same set?

Answer 1. Yes. See Theorem 2 below.

Question 2 (K. Hickin [1]). Does there exist a faithful sharp relatively-homogeneous group that is not locally finite?

Answer 2. Yes. See Theorem 4 below.

Other connected results are also stated below. Question 1 arose while seeking possible generalizations of some theorems of Mekler and Truss on embeddings of infinite groups 
(cf. [2]). Actually, Answer 1 was given independently by Truss and the present author. Theorem 1 was conjectured by P. M. Neumann as a possible generalization of a more restricted theorem proved by the author. Question 2 was posed in Hickin's study [1] on infinite homogeneous permutation groups. One notes that Theorem 3 is an immediate consequence of Theorem 2 since the embedding in Theorem 2 is proper. Theorem 3 is also a partial answer to P. M. Neumann's question on maximal sharp groups which always exist by the Maximal Principle of Set Theory.

The main results proved below are the following:

Theorem 1. Let $G, H$ be countable sharp groups acting on a countably infinite set $\Omega$. Then there exists a permutation $y$ of $\Omega$ such that

(i) $\left\langle G, y H y^{-1}\right\rangle$ is a sharp group, and

(ii) $\left\langle G, y H y^{-1}\right\rangle$ is the free product $G^{*} y H y^{-1}$ of $G$ and $\left.y H^{-1}\right\}$

Theorem 2. Given a countable sharp permutation group $(G, \Omega)$ with $\Omega$ countably infinite, $G$ can be properly embedded in a countable sharp, highly transitive permutation group $\left(G^{*}, \Omega\right)$ acting on the same set $\Omega$.

Theorem 3. Every maximal sharp group on a countably infinite set is uncountable.

Theorem 4. There exists a faithful countably infinite permutation group $(G, \Omega)$ of countably infinite degree such that

(i) $(G, \Omega)$ is not locally finite,

(ii) $(G, \Omega)$ is sharp and relatively homogeneous, and

(iii) the orbits of every finitely generated subgroup of $G$ are finite.

The proof of Theorem 4 is in Section 4, while the others are proved in Section 3. Section 2 contains some preliminaries. Where the proof of a theorem is long, the central idea in the proof is given immediately before the proof. Outlines are also given in some proofs to indicate their directions.

\section{Preliminaries}

Definitions. Let $A_{1}, A_{2}, \ldots, A_{n}$ be infinite sets.

1. The projections $\rho_{1}, \rho_{2}$ of $A_{1} \times A_{2}$ are defined by

$$
\begin{aligned}
\rho_{1}: A_{1} \times A_{2} & \rightarrow A_{1} & \rho_{2}: A_{1} \times A_{2} & \rightarrow A_{2} \\
\left(a_{1}, a_{2}\right) & \mapsto a_{1}, & \left(a_{1}, a_{2}\right) & \mapsto a_{2}
\end{aligned}
$$

for every $\left(a_{1}, a_{2}\right) \in A_{1} \times A_{2}$.

2. A curve in $A_{1} \times A_{2}$ is a non-empty subset $S$ of $A_{1} \times A_{2}$ such that for $i=1$ or 2 , the restriction of the projection $\rho_{i}$ to $S$ is injective. 
Note 2.1. This means that for points on a curve, the values of the first components determine the values of the second or vice-versa.

Note 2.2. Observe that the subset of $A_{1} \times A_{2}$ consisting of elements with equal components is a curve in $A_{1} \times A_{2}$.

Lemma 5. Let $\Omega$ be an infinite set and $x$ a permutation of $\Omega$ with cofinite support; i.e. the set $\{\alpha \in \Omega \mid \alpha x=\alpha\}$ is finite or empty. Let $\xi_{1}, \ldots, \xi_{p}$ be fixed elements of $\Omega$ and $A_{1}, A_{2}$ fixed infinite subsets of $\Omega$. If

$$
S:=\left\{\left(\gamma_{1}, \gamma_{2}\right) \mid \gamma_{1} \in\left\{\gamma_{1}, \gamma_{2}, \xi_{1}, \ldots, \xi_{p}\right\} x\right\}
$$

then $S$ is a finite union of curves in $A_{1} \times A_{2}$.

Proof of Lemma 5. If the hypotheses of the lemma hold, then we have the following possibilities: either

(i) $\gamma_{1}=\xi_{i} x$ for some $i=1, \ldots, p$;

or (ii) $\gamma_{1}=\gamma_{2} x$

or (iii) $\gamma_{1} x=\gamma_{1}$.

It is clear that cases (i) and (ii) lead to a finite union of curves in $A_{1} \times A_{2}$. Case (iii) also does because the support of $x$ is cofinite by hypothesis.

Lemma 6. Let $N$ be a positive integer. If $A_{1}, A_{2}$ are infinite sets and $S_{1}, \ldots, S_{N}$ are curves in $A_{1} \times A_{2}$, then

$$
\left(A_{1} \times A_{2}\right) \backslash \bigcup_{i=1}^{N} S_{i} \quad \text { is infinite. }
$$

Proof of Lemma 6. Let $S_{1}, \ldots, S_{N}$ be as in the hypotheses of lemma. Suppose the restriction of $\rho_{2}$ to $S_{1}$ is injective. Define

$$
\begin{aligned}
& C\left(S_{1}, \phi\right):=\left\{y \in A_{2} \mid\left(\forall a_{1} \in A_{1}\right)\left(\left(a_{1}, y\right) \notin S_{1}\right)\right\} \\
& C\left(S_{1}, D\right):=\left\{y \in A_{2} \mid(\exists d \in D)\left((d, y) \in S_{1}\right)\right\}
\end{aligned}
$$

for every subset $D$ of $A_{1}$. It follows from the definition of a curve that if $D_{1} \cap D_{2}=\phi$, then $C\left(S_{1}, D_{1}\right) \cap C\left(S_{1}, D_{2}\right)=\phi$. If $C\left(S_{1}, \phi\right)$ is infinite, then put $A_{11}:=A_{1}, A_{21}:=C\left(S_{1}, \phi\right)$. Then we have $\left(A_{11} \times A_{21}\right) \cap S_{1}=\phi$ with $A_{11}, A_{21}$ being infinite sets and $S_{2} \cap\left(A_{11} \times A_{21}\right), \ldots, S_{N} \cap\left(A_{11} \times A_{21}\right)$ being curves in $A_{11} \times A_{21}$.

Suppose $C\left(S_{1}, \phi\right)$ is not infinite. Then $C\left(S_{1}, A_{1}\right)$ is infinite since $A_{2}$ is. Choose a subset $E$ of $A_{1}$ such that both $E$ and $A_{1} \backslash E$ are infinite. Then it follows that one of $C\left(S_{1}, E\right)$, $C\left(S_{1}, A_{1} \backslash E\right)$ is infinite. Let $A_{11}$ be $E$ or $A_{1} \backslash E$ such that $C\left(S_{1}, A_{1} \backslash A_{11}\right)$ is infinite; and put $A_{21}:=C\left(S_{1}, A_{1} \backslash A_{11}\right)$. It then follows that $\left(A_{11} \times A_{21}\right) \cap S_{1}=\phi$ - the same result as in the last paragraph. Clearly a similar result will hold if the restriction of $\rho_{1}$ to $S_{1}$ is injective. 
The procedure above involves $A_{1} \times A_{2}$ and $S_{1}$. We can now apply a similar procedure to $A_{11} \times A_{21}$ and $S_{2}$ to obtain that $\left(A_{12} \times A_{22}\right) \cap\left(S_{1} \cup S_{2}\right)=\phi$ for some infinite sets $A_{12} \subseteq A_{11}, A_{22} \subseteq A_{21}$. After $N$ such steps, we then have the assertion of the lemma.

\section{Embeddings in highly transitive groups}

Proof of Theorem 1. The theorem is trivial if either $G$ or $H$ equals $\{1\}$. So we assume this is not so.

For now, let $y$ be a symbol. Let

$$
\omega_{1}, \omega_{2}, \omega_{3}, \ldots
$$

be an (countable) enumeration of all the words of the form:

$$
\omega_{i}(y):=\left(y h_{i 1} y^{-1}\right) g_{i 1}\left(y h_{i 2} y^{-1}\right) g_{i 2} \ldots\left(y h_{i n(i)} y^{-1}\right) g_{i n(i)}
$$

where

$$
g_{i j} \in G \backslash\{1\}, \quad h_{i j} \in H \backslash\{1\}
$$

for all $i$ and $1 \leqq j \leqq n(i)$. To prove the theorem, it suffices to construct a map $y$ satisfying (1.1)(i) and such that $\omega_{i}(y)$ has cofinite support for every $i$. This is because every element of $\left\langle G, y H y^{-1}\right\rangle$ is a conjugate of some $\omega_{i}$ or some element in $G \cup y H y^{-1}$.

Outline. The procedure we follow is to construct approximations $y_{1}, y_{2}, \ldots$ to $y$ such that $y_{i+1}$ is an extension of $y_{i}$ and such that $\mu \omega_{j} \neq \mu$ for all $\mu$ outside the finite domain of $y_{j-1}$. The latter property makes strong use of sharpness of $G$ through Lemma 5 . The construction also ensures that the unions of the domains and ranges of the partial maps $y_{i}$ do not miss out any element of $\Omega$.

Let $\Omega:=\left\{\alpha_{0}, \alpha_{1}, \alpha_{2}, \ldots\right\}$ be an enumeration of $\Omega$ as a sequence. More precisely, we want to construct $y_{1}, y_{2}, \ldots$ such that for each $i$ :

(i) $\mathscr{D}\left(y_{i}\right) \subset \Omega, \mathscr{R}\left(y_{i}\right) \subset \Omega$ where $\mathscr{D}\left(y_{i}\right), \mathscr{R}\left(y_{i}\right)$ denote the domain and range of $y_{i}$ and are finite sets.

(ii) $\alpha_{0}, \alpha_{1}, \ldots, \alpha_{i-1} \in \mathscr{D}\left(y_{i}\right)$ $\alpha_{0}, \alpha_{1}, \ldots, \alpha_{i} \in \mathscr{R}\left(y_{i}\right)$.

(iii) $y_{i-1}=y_{i} \mid \mathscr{D}_{\left(y_{i-1}\right)}$; that is $y_{i}$ is an extension of $y_{i-1}$.

(iv) $\mu \omega_{j}\left(y_{i}\right) \neq \mu \forall \mu \in \mathscr{D}\left(y_{i}\right) \backslash\left(\mathscr{D}\left(y_{j-1}\right)\right)$ and $1 \leqq j \leqq i$. The inequality includes the possibility that the LHS may not be well-defined. For uniformity, we define $\mathscr{D}\left(y_{0}\right):=\phi$. $=\phi$.

(v) $y_{i}$ is a map of form: $\beta_{i 1} \mapsto \beta_{i 2} \mapsto \beta_{i 3} \mapsto \cdots \mapsto \beta_{i m(i)}$.

\section{Construction of $y_{1}$}

Let $\gamma_{1}, \gamma_{-1}$ be variables which take distinct values in $A_{1}:=\left\{\alpha_{2}, \alpha_{3}, \ldots\right\}$. Then $\gamma:=$ 
$\left(\gamma_{-1}, \gamma_{1}\right) \in \mathscr{A}_{1}:=A_{1}^{2}$. Let $y_{1}(\gamma)$ be the function:

$$
\gamma_{-1} \mapsto \alpha_{0} \mapsto \gamma_{1} \mapsto \alpha_{1}
$$

Consider the system of equations:

$$
\mu \omega_{1}\left(y_{1}(\gamma)\right)=\mu, \quad \mu \in\left\{\gamma_{-1}, \alpha_{0}, \gamma_{1}\right\}
$$

\section{Outline}

We show next that the set of all $\gamma$ in $\mathscr{A}_{1}$ for which any one of (3.3) holds is contained in the union of a finite number of curves of $\mathscr{A}_{1}$.

Suppose $\alpha_{0} \omega_{1}\left(y_{1}(\gamma)\right)=\alpha_{0}$. Then,

$$
\gamma_{1} h_{11} y_{1}^{-1} g_{11}\left(y_{1} h_{12} y_{1}^{-1}\right) g_{12} \ldots\left(y_{1} h_{1 \mathrm{n}(1)} y_{1}^{-1}\right) g_{\ln (1)}=\alpha_{0}
$$

This implies that $\gamma_{1} h_{11} \in \mathscr{R}\left(y_{1}\right)$ which is $\left\{\alpha_{0}, \gamma_{1}, \alpha_{1}\right\}$. Using Lemma 5 , we deduce that the collection of $\gamma$ satisfying (3.3) for $\mu=\alpha_{0}$ is at most a union of a finite number of curves in $\mathscr{A}_{1}$.

For $\mu=\gamma_{-1}$ and $\mu=\gamma_{1}$, equation (3.3) becomes respectively

$$
\begin{aligned}
& \alpha_{0} h_{11} y_{1}^{-1} g_{11} \ldots y_{1} h_{\ln (1)} y_{1}^{-1} g_{\ln (1)}=\gamma_{-1} \\
& \alpha_{1} h_{11} y_{1}^{-1} g_{11} \ldots y_{1} h_{\ln (1)} y_{1}^{-1} g_{\ln (1)}=\gamma_{1}
\end{aligned}
$$

In each of the two cases,

$$
\gamma_{i} \in\left\{\gamma_{-1}, \alpha_{0}, \gamma_{1}\right\} g_{\ln (1)}
$$

for some $\gamma_{i}, i=1,-1$. This is so because the element that comes before $g_{\ln (1)}$ on the LHS of (3.4) is in the domain of $y_{1}$. We conclude as in the last paragraph that only points on a finite number of curves of $\mathscr{A}_{1}$ satisfy (3.3) for any of the two cases.

Hence the collection of $\gamma$ satisfying any equation in (3.3) (which is a finite system of equations) is contained in a finite union of curves. With the use of Note 2.2 and Lemma 6 , we then choose some $\gamma$ (with distinct components) in the complement of this union; and for simplicity, we put $y_{1}:=y_{1}(\gamma)$. Thus, $y_{1}$ violates every equation in (3.3).

\section{Construction of $y_{s+1}$, assuming $y_{s}$ is known}

Assume $y_{s}$ satisfies $3.2(\mathrm{i})-(\mathrm{v})$ for $i=s$. Let $\left\{\delta_{0}, \delta_{1}, \delta_{2}, \ldots\right\}$ be a subsequence of $\left\{\alpha_{0}, \alpha_{1}, \alpha_{2}, \ldots\right\}$ such that $\left\{\delta_{0}, \delta_{1}, \ldots\right\}=\Omega \backslash \mathscr{R}\left(y_{s}\right)$. Suppose $\delta_{j}=\alpha_{m(j)}$. By definition of subsequence and since $\left\{\alpha_{i}\right\}$ has distinct terms, we have that $k<j$ if and only if $m(k)<m(j)$. This with 3.2(ii) for $i=s$ implies $\alpha_{s+1} \in \mathscr{R}\left(y_{s}\right)$ or $\alpha_{s+1}=\delta_{0}$. Put

$$
A_{s+1}:=\Omega \backslash\left(\mathscr{D}\left(y_{0}\right) \cup\left\{\delta_{0}\right\}\right) \text { and } \mathscr{A}_{s+1}:=A_{s+1}^{2} \text {. }
$$


As done for $y_{1}$, let $\gamma:=\left(\gamma_{-1}, \gamma_{1}\right)$ be a variable which can take values in $A_{s+1}$ and with distinct components. With the notations in 3.2(v), let

$$
y_{s+1}(\gamma): \gamma_{-1} \mapsto \beta_{s 1} \mapsto \beta_{s 2} \mapsto \cdots \mapsto \beta_{s m(s)} \mapsto \gamma_{1} \mapsto \delta_{0} .
$$

It should be clear from (3.2)(v), (ii) for $i=s$, that $\alpha_{s} \in \mathscr{D}\left(y_{s+1}(\gamma)\right)$. Consider the system of equations:

$$
\mu \omega_{j}\left(y_{s+1}(\gamma)\right)=\mu, \quad \mu \in \mathscr{D}\left(y_{s+1}\right), \quad \text { and } \quad 1 \leqq j \leqq s+1 \ldots
$$

Let $\omega_{j}$ be any word within the given range in (3.5), and let $\mu=\beta_{s m(s)}$. The arguments similar to those used for (3.3) when $\mu=\alpha_{0}$ show that the solution set for (3.5) when $\mu=\beta_{s m(s)}$ is at most a finite union of curves in $\mathscr{A}_{s+1}$. We also arrive at the same result if $\mu \in\left\{\gamma_{-1}, \gamma_{1}\right\}$ and if we use arguments like those used for (3.3) where $\mu=\gamma_{1}$.

For a fixed $\omega_{j}, 1 \leqq j \leqq s$, let $\mu \in \mathscr{D}\left(y_{s}\right)-\mathscr{D}\left(y_{j-1}\right)$. By inductive hypothesis (3.2)(iv) for $i=s$, either $\mu \omega_{j}\left(y_{s}\right)$ is undefined or it is defined and unequal to $\mu$. If the latter occurs, then $\mu \omega_{j}\left(y_{s+1}(\gamma)\right)=\mu \omega_{j}\left(y_{s}\right) \neq \mu$ for any $\gamma$. If on the other hand, $\mu \omega_{j}\left(y_{s}\right)$ is undefined, then in order for

$$
\mu \omega_{j}\left(y_{s+1}(\gamma)\right)=\mu
$$

to hold for the stipulated range of $\mu$ in this paragraph, an element

$$
\nu \in\left(\mathscr{D}\left(y_{s+1}(\gamma)\right) \backslash \mathscr{D}\left(y_{s}\right)\right) \cup\left(\mathscr{R}\left(y_{s+1}(\gamma) \backslash \mathscr{R}\left(y_{s}\right)\right)\right.
$$

has been used in the computation of the LHS of (3.6). This means that some $\gamma_{i}$ occurs before or after some $g_{j k}$ or $h_{j k}$. Using Lemma 5, we then conclude that the solution set of (3.6) is at most a finite union of curves in $\mathscr{A}_{s+1}$. This result and the result in the last paragraph, together with Note 2.2 and Lemma 6 , show that we can pick $\gamma \in \mathscr{A}_{s+1}$ with distinct components such that (3.2)(iv) holds for $i=s+1$. The method of construction shows clearly that (3.2)(i), (ii), (iii), (v) all hold for $i=s+1$. This ends the inductive step from $i=s$ to $i=s+1$. We then conclude that (3.2)(i)- (v) holds for all $i$.

Now, using definition of a function on $\Omega$ as a special subset of $\Omega \times \Omega$, we let

$$
y:=\bigcup_{i=1}^{\infty} y_{i}
$$

From (3.2)(i)-(iii), we then have that $y: \Omega \rightarrow \Omega$. From (3.2)(iv), (v), we have that, for every $j$,

$$
\mu \omega_{j}(y) \neq \mu, \quad \forall \mu \in \Omega \backslash \mathscr{D}\left(y_{j-1}\right)
$$

and that $y$ is a single infinite cycle on $\Omega$. As stated at the beginning of the proof of this theorem, (3.7) implies the assertion of the theorem, since every element of $\mathrm{G} * y \mathrm{Hy}^{-1}$ is a conjugate of some $\omega_{i}$ or some element of $\mathrm{G} \cup y \mathrm{Hy}^{-1}$. 
Proof of Theorem 2. Let $\Omega:=\left\{\beta_{1}, \beta_{2}, \ldots\right\}$ be an enumeration of $\Omega$. For every $i$, $i=0,1,2, \ldots$ let $h_{i}$ be a permutation that permutes $\left\{\beta_{i+1}, \beta_{i+2}, \ldots\right\}$ in one infinite cycle and fixes the rest. Note that $\left\langle h_{i}\right\rangle$ is sharp for every $i$. By Theorem 1 , there exists $y_{1}$ such that $\left\langle h_{0}, y_{1} h_{1} y_{1}^{-1}\right\rangle$ is sharp and free. When the theorem is applied to the groups $\left\langle h_{0}, y_{1} h_{1} y_{1}^{-1}\right\rangle$ and $\left\langle h_{2}\right\rangle$ we see that there exists $y_{2}$ such that $\left\langle h_{0}, y_{1} h_{1} y_{1}^{-1}, y_{2} h_{2} y_{2}^{-1}\right\rangle$ is sharp and free. In general we shall have that $\left\langle h_{0}, y_{1} h_{1} y_{1}^{-1}, y_{2} h_{2} y_{2}^{-1}, y_{3} h_{3} y_{3}^{-1}, \ldots\right\rangle$ is sharp and free for some $y_{1}, y_{2}, y_{3}, \ldots$. Put $x_{0}:=h_{0}, x_{i}:=y_{i} h_{i} y_{i}^{-1}, i=1,2, \ldots$. Note that for each $i,\left\langle x_{i}\right\rangle$ is transitive on $\left\{\beta_{i+1}, \beta_{i+2}, \ldots\right\} y_{i}^{-1}$. Hence $H:=\left\langle x_{0}, x_{1}, x_{2}, \ldots\right\rangle$ is sharp and highly transitive. By Theorem 1 again, there exists a permutation $y$ on $\Omega$ such that $G * y H y^{-1}$ is sharp and highly transitive. Since $y H y^{-1} \neq 1$ and since $G * y H y^{-1}$ is a free product of $G$ and $y H y^{-1}$, then $G * y H y^{-1}$ contains $G$ properly.

As stated in the introduction, Theorem 3 is an immediate consequence of Theorem 2.

\section{Idea of proof of Theorem 4}

Both the domain and the group are extended inductively so that we have a sequence $\left(G_{1}, \Sigma_{1}\right),\left(G_{2}, \Sigma_{1} \cup \Gamma_{2}\right),\left(G_{3}, \Sigma_{1} \cup \Gamma_{2} \cap \Gamma_{3}\right), \ldots$ where $G_{1}, G_{2}, \ldots, \Sigma_{1}, \Gamma_{2}, \Gamma_{3}, \ldots$ are all finite. An appropriate limit $(G, \Omega)$ is taken as the desired permutation group.

The extensions have the property that the extension of every nonidentity group element is non-identity. This, with another property that the action of the extensions is always defined as a right-translation in a regular representation, makes $(G, \Omega)$ sharp. Moreover, the orders of the extensions of the group elements are made to increase at each step. This makes the order of some elements infinite and thus makes $(G, \Omega)$ nonlocally finite. To ensure relative homogeneity, we also arrange that the extensions of commutative group elements are commutative.

\section{Proof of Theorem 4.}

\section{Construction of $(G, \Omega)$}

For now, let $\Sigma$ be any non-empty finite set and let $S$ be $\operatorname{Sym}(\Sigma)$, the set of all permutations of $\Sigma$. Also, let $A(S)$ be the group generated by new symbols $a(s)$ for $s \in S$ subject to the relations $[a(s), a(t)]=1, a(s)^{|S|}=1$. Let

$$
\begin{gathered}
\Gamma:=S \times A(S) \\
\Sigma^{*}:=\Sigma \cup(S \times A(S)) .
\end{gathered}
$$

Here, $[x, y]$ denotes the commutator $x^{-1} y^{-1} x y$, and $|S|$ denotes the number of elements in $S$. For each $s \in S$, define $r(s)$, to be right multiplication by $(s, a(s))$ acting faithfully on $S \times A(S)$, and put

$$
s^{*}:=(s, r(s)) \in \operatorname{Sym}\left(\Sigma^{*}\right)
$$


To construct $(G, \Omega)$, let $\Sigma_{1}$ be any non-empty finite set. Using the notations above we define

$$
\begin{gathered}
\Sigma_{n}:=\Sigma_{n-1}^{*}, \\
\Gamma_{n}:=\Sigma_{n-1}^{*} \backslash \Sigma_{n-1} \\
\Omega:=\bigcup_{1}^{\infty} \Sigma_{n} \\
G_{n}:=\operatorname{Sym}\left(\Sigma_{n}\right) .
\end{gathered}
$$

The group $G$ is a subgroup of $\operatorname{Sym}(\Omega)$ defined by

$$
\left.G:=\left\langle\mathbf{s}:=\left(s_{(0)}, s_{(1)}, s_{(2)}, \ldots\right)\right| s_{(0)} \in G_{n} \text { for some } n\right\rangle .
$$

Here,

$$
\begin{gathered}
s_{(1)}:=r\left(s_{(0)}\right) \\
s_{(2)}:=r\left(\left(s_{(0)}, s_{(1)}\right)\right) \\
s_{(3)}:=r\left(s_{(0)}, s_{(1)}, s_{(2)}\right) \\
:=r\left(\left(\left(s_{(0)}, s_{(1)}\right), s_{(2)}\right)\right) \\
s_{(n)}:=r\left(s_{(0)}, s_{(1)}, \ldots, s_{(n-1)}\right) .
\end{gathered}
$$

Some properties of $(G, \Omega)$

Let $\omega, \omega_{1}, \omega_{2}$ denote arbitrary words. We wish to note some properties of $(G, \Omega)$ constructed above. For every natural number $p$, the following are true:

(a)

$$
\left|\Sigma_{p}\right|<\left|\Sigma_{p+1}\right|<\infty ; \quad \Sigma_{p} \subset \Sigma_{p+1} .
$$

These are obvious from the construction above.

(b) The permutation group $(G, \Omega)$ is faithful. This is because each $s_{(i)}$ is defined as a permutation.

(c) For every $s_{1}, \ldots, s_{k} \in \operatorname{Sym}\left(\Sigma_{p}\right)$,

$$
\omega\left(s_{1}, \ldots, s_{k}\right) \neq 1 \Rightarrow \operatorname{supp}_{\Gamma_{p+1}} \omega\left(r\left(s_{1}\right), \ldots, r\left(s_{k}\right)\right)=\Gamma_{p+1} .
$$

To see this, suppose $\omega\left(s_{1}, \ldots, s_{k}\right) \neq 1$. A typical element of $\Gamma_{p+1}$ is

$$
\left(s, u\left(a\left(s_{i 1}\right), a\left(s_{i 2}\right), \ldots, a\left(s_{i m}\right)\right)\right)
$$


where $u$ is a word and $m$ is a natural number. Then for every such element, we have that the group action

$$
\begin{aligned}
\left(\left(s, u\left(a\left(s_{i 1}\right), a\left(s_{i 2}\right), \ldots, a\left(s_{i m}\right)\right)\right) \omega\left(r\left(s_{1}\right), \ldots, r\left(s_{k}\right)\right)\right. & =\left(s \omega\left(s_{1}, \ldots, s_{k}\right), \bar{a}\right) \quad \text { for some } \bar{a} \in A\left(G_{p}\right) \\
& \neq(s, a(s)) \text { since } \omega\left(s_{1}, \ldots, s_{k}\right) \neq 1 .
\end{aligned}
$$

(d) For every $s_{1}, \ldots, s_{l} \in \operatorname{Sym}\left(\Sigma_{p}\right), \omega_{1}\left(s_{1}, \ldots, s_{l}\right)$ commutes with $\omega_{2}\left(s_{1}, \ldots, s_{l}\right)$ if and only if $\omega_{1}\left(r\left(s_{1}\right), \ldots, r\left(s_{l}\right)\right)$ commutes with $\omega_{2}\left(r\left(s_{1}\right), \ldots, r\left(s_{l}\right)\right)$ where $\omega_{1}, \omega_{2}$ are any words.

We show this briefly. Now, by definition, the permutation $\omega_{i}\left(r\left(s_{1}\right), \ldots, r\left(s_{l}\right)\right)$ of $\Gamma_{p+1}$ is a right multiplication by $\left(\omega_{i}\left(s_{1}, \ldots, s_{l}\right), \omega_{i}\left(a\left(s_{1}\right), \ldots, a\left(s_{l}\right)\right)\right)$ for $i=1,2$. The second components in the last expression for $i=1,2$ commute in their action on $A\left(G_{p}\right)$ since by definition they are elements of an abelian group acting regularly on the same abelian group. The first components commute in their regular action on $G_{p}$ since they commute as elements of $G_{p}$.

(e) For any $s \in G_{p} \backslash\{1\}$, the element $(s, r(s))$ has order $\left|G_{p}\right|$ as an element of $G_{p+1}$.

To show this, let $M=\left|G_{p}\right|$. If $s \neq 1$, then $a(s)$ has order $M$ by definition of $A\left(G_{p}\right)$. Moreover, $s^{M}=1$ since $s \in G_{p}$. Therefore, the order of $r(s)$ is $M$ and so the order of $(s, r(s))$ is $M$.

\section{Conclusion}

We verify now that the properties listed in the theorem are satisfied by $(G, \Omega)$.

Non-local finiteness. By (4.1) and (4.5) for every $p$, we observe that every $s$ in $G \backslash\{1\}$ has infinite order. Hence $(G, \Omega)$ is not locally finite.

Sharpness. Suppose $\omega:=\omega\left(\mathbf{s}_{1}, \mathbf{s}_{2}, \ldots, \mathbf{s}_{m}\right) \neq 1 \quad$ where $\mathbf{s}_{i}=\left(s_{i(0)}, s_{i(1)}, \ldots\right), \quad i=1, \ldots, m$. Suppose also $s_{i(0)} \in G_{p_{i}}$ Then, from (4.1) and definition of $\Omega$, there exists $k$ such that $k>\max \left\{p_{1}, \ldots, p_{m}\right\}$ and the restriction $\left.\omega\right|_{\Sigma_{k}}$ of $\omega$ to $\Sigma_{k}$ is not the identity. By (4.3) for all $p$, we observe that $\left.\omega\right|_{\Gamma_{k+1}}$ has support equal to $\Gamma_{k+1}$. Repeated application of (4.1) shows that the fixed points of $\omega$ are all contained in $\Sigma_{k}$. Hence support of $\omega$ is cofinite.

Relative Homogeneity. Suppose a subgroup $B$ of $G$ is generated by words

$$
\omega_{j}\left(\mathbf{s}_{1}, \ldots, \mathbf{s}_{m}\right), \quad j=1, \ldots, k
$$

where $k, m$ are natural numbers. In this notation, we allow the possibility that a word $\omega_{i}$ may not depend on all the arguments listed. Using the notations in the last paragraph, suppose $s_{i(0)} \in G_{p_{i}}$. Let $M:=\max \left\{p_{1}, \ldots, p_{m}\right\}$. Then $\Sigma_{M}, \Gamma_{M+1}, \Gamma_{M+2}, \ldots$ are all unions of orbits of $B$. Let $f$ be a $B$-isomorphism connecting orbits $\Delta_{1}, \ldots, \Delta_{t}$ of $B$ with $t$ finite. 
Since each orbit of $B$ is finite, we therefore have that $\Delta_{1}, \ldots, \Delta_{t}$ are subsets of $\Sigma_{q}$ for some fixed $q>M$. It is a well-known fact that every such isomorphism acting on the finite set $\Sigma_{q}$ can be extended to a permutation $f$ in $\operatorname{Sym}\left(\Sigma_{q}\right)$ which is a $B$-isomorphism. By the construction of $(G, \Omega)$ above, there exists some $s$ in $G$ such that $s \mid \Sigma_{q}=\bar{f}$. Property (4.4) ensures that $s$ commutes with every element of $B$. Thus, $(G, \Omega)$ is relatively homogeneous.

Finitely generated subgroups. The arguments in the last paragraph show that if a subgroup $B$ of $G$ is generated by a finite number of words $\omega_{i}$, then there exists a natural number $M$ such that $\Sigma_{M}, \Gamma_{M+1}, \Gamma_{M+2}, \ldots$ are all unions of orbits of $B$. Since $\Sigma_{m}, \Gamma_{m+1}, \Gamma_{m+2}, \ldots$ are all finite then (1.2)(iii) holds.

Acknowledgements. This research was undertaken while the author was under the Royal Society Fellowship for Developing Countries. The author is grateful to them for the opportunity they provided, and is highly indebted to Peter M. Neumann who supervised and prompted the work.

\section{REFERENCES}

1. K. Hickin, Some results on homogeneous permutation groups, preprint (April, 1985).

2. J. K. Truss, Embeddings of Infinite Permutation Groups, Proceedings of Groups-St Andrews 1985 (London Math. Soc. Lecture Notes 121, Cambridge University Press 1986), 335-351.

Department of Mathematics

Bowling Green State University

Bowling GreEn

Онго 43403

U.S.A. 